\title{
Adenocarcinoma oncocítico oxifílico de endometrio: reporte de caso
}

\author{
Ernesto García Ayala ${ }^{1}$, Diana Katherine Sandoval Martínez¹, Juan Fernando Calvo \\ Castellanos ${ }^{a}$, Juan David Lara Lozano ${ }^{a}$. \\ ${ }^{1}$ Departamento de Patología, Facultad de Salud, Universidad Industrial de Santander, Bucaramanga, Colombia.
}

a Alumno, Escuela de Medicina, Universidad Industrial de Santander.

\section{RESUMEN}

El cáncer de endometrio es la patología maligna más frecuente del tracto genital femenino, con una incidencia del $3,2 \%$ en Latinoamérica, y que según sus características histológicas permite clasificar las lesiones en tipo I o II. Entre las variantes de esta última se encuentra el adenocarcinoma oxifílico. Se presenta caso de una paciente de 77 años con cuadro de hemorragia uterina anormal y dolor pélvico crónico, que posterior a estudios complementarios es llevada a histerectomía ampliada, en su estudio histopatológico final se encuentra lesión compuesta por células epiteliales con abundante citoplasma intensamente acidofílico con núcleos pleomórficos e hipercromáticos dispuestos en un patrón arquitectural de predominio sólido, positivo para CK7, CK14 y parcialmente positivo para Bcl2, diagnosticándose adenocarcinoma oxif́lico de endometrio. Este caso resulta importante debido al poco número de reportes a nivel mundial y por ser el primero encontrado en Colombia.

\section{PALABRAS CLAVE: Endometrio, adenocarcinoma, oxifílico, oncocítico}

\section{SUMMARY}

Endometrial cancer is the most frequent malignant pathology of the female genital tract, with an incidence of $3.2 \%$ in Latin America. Histological characteristics lead to classify this lesions in type I or II where oxyphilic adenocarcinoma is included as a variant of the last group. This is a case report of a 77 years old patient with clinical symptoms of abnormal uterine bleeding and chronic pelvic pain thatafter complementary studies is carried to extended hysterectomy; further histopathological study showed a lesion formed by epithelial cells with abundant intensely acidophilic cytoplasm with pleomorphic and hyperchromatic nuclei arranged in a predominantly solid architectural pattern, positive for CK14, CK7 and partially positive for Bcl2, diagnosing endometrial oxyphilic adenocarcinoma. This case is remarkable important because of the small number of reports worldwide and being the first found in Colombia.

\section{KEY WORDS: Endometrium, adenocarcinoma, oxyphilic, oncocytic}

\section{INTRODUCCIÓN}

El cáncer de endometrio representa un $7 \%$ de todos los tumores infiltrantes de la mujer. En los países subdesarrollados los factores de riesgo son infrecuentes y el carcinoma endometrial es de baja prevalencia, pero con mortalidad alta (1-3). Para Latinoamérica, la incidencia es del 3,2\% y la mortalidad del 2,3\%; en Colombia la incidencia es de $2 \%$ y $0,9 \%$ de mortalidad. Según el estudio de 
Uribe y cols (4), en nuestro medio el $91,1 \%$ de las lesiones invasivas del cuerpo uterino corresponden a adenocarcinomas endometriales, siendo el 3,7\% de los tumores que afectan a las mujeres y se ubica en octavo lugar de incidencia general. Según sus características histológicas el adenocarcinoma endometrial se clasifica en tipo I y II.

Los adenocarcinomas tipo I se asocian a estimulación estrogénica alta y persistente o a disminución de niveles de progesterona (2,3,5-7). Dentro de los factores de riesgo se encuentran la edad avanzada, la menarquia temprana y la menopausia tardía, los ciclos anovulatorios, la nuliparidad $(3,5,6,8,9)$, y la obesidad que conduce a producción sostenida de estrógenos no balanceada por progesterona, reducción en la síntesis de citoquinas como leptina y adipoleptina (8-10). También se asocian a tumores de ovario productores de estrógenos y síndrome de ovario poliquístico $(3,9)$. Desde el punto de vista genético esta categoría presenta mutación en el oncogén KRAS2 y el gen supresor de tumor PTEN, defectos en los genes de reparación del ADN y un cariotipo diploide $(2,3,6,7,8,11,12)$.

El adenocacarcinoma tipo II no se liga a exposición estrogénica y se observa con mayor frecuencia en mujeres afroamericanas, multíparas y edad avanzada $(3,5,8,9)$; los factores genéticos son representados por mutaciones en el gen PTEN y expresión de ERBB-2 (HER-2/neu) con un cariotipo no diploide $(2,3,7,8,12)$.

Estos tumores presentan variantes histológicas de acuerdo a características de las células que los componen, el adenocarcinoma oncocítico, ha sido poco descrito y recibe este nombre por sus células que recuerdan los cambios oncocíticos de las células foliculares tiroideas. El objetivo de este artículo es exponer un caso diagnosticado de este subtipo histológico y hacer una revisión de la literatura, dada la baja frecuencia de su diagnóstico y escasa información al respecto, siendo este el primer caso reportado en Colombia y Latinoamérica.

\section{Caso clínico}

Mujer de 77 años con cuadro de hemorragia uterina anormal y dolor pélvico crónico, con ecografía transvaginal que evidencia un endometrio engrosado. Se toma biopsia que reporta adenocarcinoma endometrial y es programada para histerectomía ampliada, salpingooforectomía bilateral, linfadenectomía regional y omentectomía. El análisis macroscópico mostró distorsión arquitectural del útero por lesión tumoral infiltrante difusa que compromete la cavidad endometrial y se extiende en el espesor de la pared miometrial a la cual infiltra (Figura 1). El estudio histopatológico evidencia tumor de origen epitelial constituido predominantemente por elementos celulares grandes, en los cuales destaca abundante citoplasma notable e intensamente acidofílico, con núcleos de diferente forma y tamaño, pleomórficos e hipercromáticos (grado nuclear entre II y III), dispuestos en un patrón arquitectural de predominio sólido con tendencia a la formación focal de estructuras glandulares y túbulos con ocasionales papilas periféricas (Figura 2). Se efectúa el diagnóstico de adenocarcinoma oxifílico de endometrio, de patrón macroscópico infiltrante difuso, que mide 6,0 x 4,0 $\mathrm{cm}$ en sus dimensiones mayores cruzadas, llega a 2 $\mathrm{mm}$ de la serosa uterina de una pared que tiene 4,0 $\mathrm{cm}$ de espesor y compromete hasta el istmo uterino sin invasión vascular sanguínea, linfática o de perineuros, la coloración de inmunohistoquímica muestra positividad celular para las citoqueratinas 7 y 14 (CK7 y CK14), con positividad parcial citoplasmática de Bcl2 (Figura 3), a su vez las células tumorales fueron negativas para CK20 y antígeno carcinoembrinario (CEA) (Figura 4), lo cual confirma el origen histológico endometrial con diferenciación oncocítica. La totalidad de los ganglios de la linfadenectomía no muestran lesión tumoral. La paciente 6 meses después del diagnóstico no ha presentado recaídas.

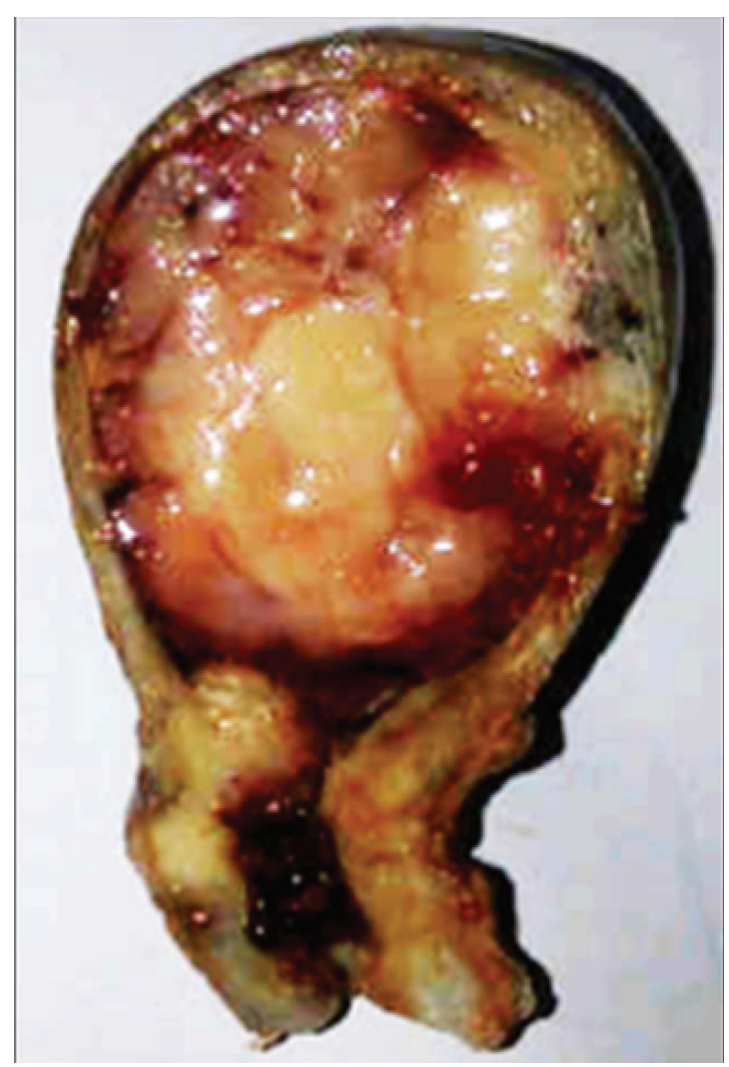

Figura 1. Imagen macroscópica de útero, en la que se observa lesión tumoral que compromete la cavidad uterina en su totalidad infiltrando su pared, la lesión es de consistencia blanda, blanco amarillenta con focos de necrosis y hemorragia. 


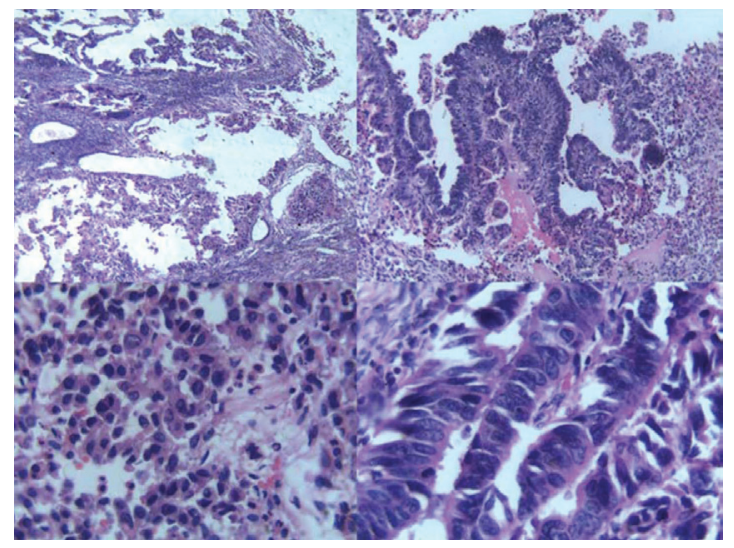

Figura 2. Imagen histológica de pared endomiometrial con lesión tumoral maligna epitelial, constituida por células grandes con abundante citoplasma acidofílico y núcleos pleomórficos e hipercromáticos (grado nuclear entre II y III), dispuestos en un patrón de predominio sólido con tendencia a la formación de túbulos con ocasionales papilas.

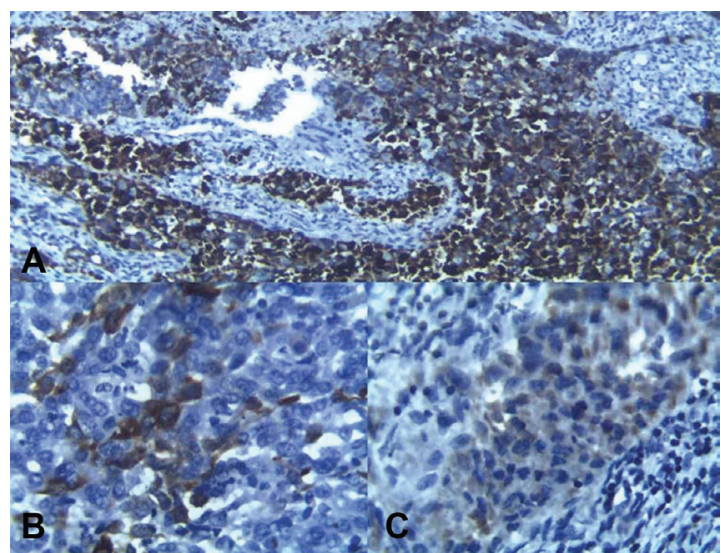

Figura 3. Marcación de inmunohistoquímica. A) CK7: positividad de las células tumorales. B) CK14: positividad de las células tumorales. C) Bcl2: positividad citoplasmatica de algunas de las células tumorales.

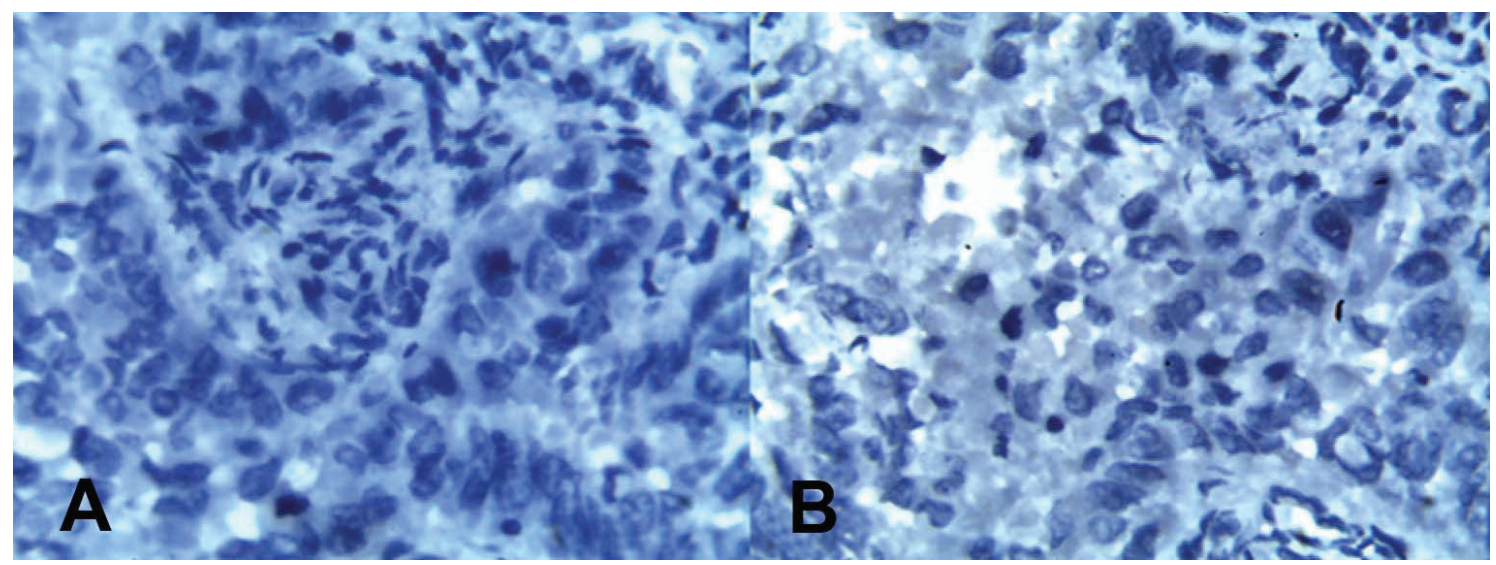

Figura 4. Marcación de inmuno histoquímica negativa en las celulas tumorales: A) CK20 B) CEA

\section{DISCUSIÓN}

El epitelio glandular del endometrio debido a sus constantes cambios mediados por hormonas, presenta con frecuencia cambios reactivos y metaplásicos, entendiéndose, estos últimos, como el reemplazo parcial por elementos celulares diferentes sin que necesariamente impliquen cambio neoplásico. El llamado cambio oncocítico o eosinofílico, se caracteriza por la presencia de intensa acidofilia citoplasmática con o sin granulaciones que puede ser observada en el epitelio endometrial normal, hiperplásico y neoplásico (13-15), el origen de este cam- bio no es claro y se ha considerado diferenciación mucinosa inmadura (16), encontrándose ausencia de componente endocrino a pesar de la expresión de TTF-1 $(13,17)$. El adenocarcinoma oncocítico de endometrio se caracteriza por estar constituido por células, que muestran marcada eosinofilia citoplasmática debido a la presencia ultraestructural de numerosas mitocondrias $(13,14,16,18)$, encontrándose en dichas células expresión de TTF-1 (14,16-18), reactividad para CK7 con negatividad de la CK20, en un patrón de presentación que se observa para la mayoría de las lesiones epiteliales de tracto genital femenino $(2,13,16,18)$, con rara 
positividad para los receptores de estrógenos y progestágenos $(16,18)$, siendo negativo para tiroglobulina dado el carácter no funcionante de estas lesiones $(13,16,18)$.

Esta variante se ha clasificado dentro de los adenocarcinomas endometriales tipo II (18) que son aquellas que no se relacionan con sobre exposición estrogénica $(2,3,7,9)$ y cuya apariencia histológica está constituida en su mayoría por células acidofílicas con arreglos arquitecturales que van desde patrones sólidos hasta formaciones papilares (18) en un patrón morfológico similar al observado en este caso (Figura 2). El análisis inmunohistoquímico demuestra origen endometrial, con positividad para CK7 y negatividad para CK20, y llama la atención la positividad para CK14 que clásicamente esta descrita para el origen tiroideo, sin embargo los casos publicados de carcinomas oncocíticos en otras localizaciones muestran aproximaciones con las lesiones tiroideas sin actividad endocrina evidente en estas células $(16,18)$. Por último el adenocarcinoma oncocítico de endometrio no ha sido reportado en Colombia ni Latinoamérica con pocos casos descritos en el mundo $(13,16,18)$, es una variante rara de adenocarcinoma endometrial que requiere distinción de otras variantes e incluso de metástasis.

\section{REFERENCIAS}

1. Ferlay J, Shin HR, Bray F, Forman D, Mathers C, Parkin DM. GLOBOCAN 2008 v2.0, Cancer Incidence and Mortality Worldwide: IARC CancerBase No. 10 [Internet]. Lyon, France: International Agency for Research on Cancer; 2010. Disponible en: http://globocan.iarc.fr.

2. Ellenson LH, Ronnett BM. Endometrial Carcinoma. In Blaustein's pathology of the female genital tract. 6th ed, Kurman RJ (Ed) New York: Springer Verlag. 2011:394-438.

3. Silverberg SG, Kurman RJ, Nogales F. Epithelial tumors and related lesions. In IARC WHO Classification of Tumors. Pathology and genetics. Tumors of the breast and female genital organs. 3ed Tavassoli FA (Ed). 2003;4: 221-32.

4. Uribe CJ, Meza EE. Incidencia de cáncer en el Área Metropolitana de Bucaramanga, 2000-2004." MedUNAB 2010;10(3):147-72.

5. Karageorgi S, Hankinson SE, Kraft P, De Vivo I. Reproductive factors and postmenopausal hormone use in relation to endometrial cancer risk in the Nurses' Health Study cohort 1976-2004. Int J Cancer 2010;126(1):208-16.
6. Hanson H, Hodgson S. Cancer genetics and reproduction. Best Pract Res Clin Obstet Gynaecol 2010;24(1):3-18.

7. Horn LC, Meinel A, Handzel R, Einenkel J. Histopathology of endometrial hyperplasia and endometrial carcinoma: an update. Ann Diagn Pathol 2007;11(4):297311.

8. Amant F, Moerman P, Neven P, Timmerman D, Van Limbergen E, Vergote I. Endometrial cancer. Lancet 2005;366(9484):491-505.

9. Felix AS, Weissfeld JL, Stone RA, Bowser R, Chivukula M, Edwards RP, Linkov F. Factors associated with Type I and Type II endometrial cancer. Cancer Causes and Control 2010;21(11):1851-6.

10. Lindemann K, Vatten LJ, Ellstrøm-Engh M, Eskild A. Body mass, diabetes and smoking, and endometrial cancer risk: a follow-up study. $\mathrm{Br} \mathrm{J}$ Cancer 2008;98(9):1582-5.

11. Turbiner J, Moreno-Bueno G, Dahiya S, Sánchez-Estevez C, Hardisson D, Prat J, Oliva E, Palacios J. Clinicopathological and molecular analysis of endometrial carcinoma associated with tamoxifen. Mod Pathol 2008;21(8):925-36.

12. Sherman ME. Theories of endometrial carcionogenesis: a multidisciplinary approach. Mod Pathol 2000;13(3):295-308.

13. Malpica A, Deavers M, Euscher E. Endometrial carcinomas. In Biopsy Interpretation Series. Biopsy Interpretation of the Uterine Cervix and Corpus. 1ed. 2010;7:177-211

14. Moritani S, Kushima R, Ichihara S, Okabe H, Hattori T, Kobayashi TK, Silverberg SG. Eosinophilic cell change of the endometrium: a possible relationship to mucinous differentiation. Mod Pathol 2005; 18(9):1243-8.

15. Nicolae A, Preda O, Nogales F. Endometrial metaplasias and reactive changes: a spectrum of altered differentiation. J Clin Pathol 2011;64:97e106.

16. Silver SA, Cheung AN, Tavassoli FA. Oncocytic metaplasia and carcinoma of the endometrium: an immunohistochemical and ultrastructural study. Int J Gynecol Pathol 1999;18(1)12-9.

17. Siami K, McCluggage WG, Ordonez NG, Euscher ED, Malpica A, Sneige N, et al. Thyroid transcription factor-1 expression in endometrial and endocervical adenocarcinomas. Am J Surg Pathol 2007;31(11):1759-63.

18. Kajiwara H, Kumaki N, Hirabayashi K, Miyazawa M, Nakamura N, Hirasawa T, Muramatsu T, Mikami M, Yasuda M, Osamura RY. A case of oncocytic carcinoma of the endometrium. Arch Gynecol Obstetrics 2009;279(5):733-8. 